\title{
Polarmetric SAR Data Compression Using Wavelet Packets in a Block Coding Scheme
}

\author{
I. Cumming, J. Wang \\ Dept. of Electrical and Computer Engineering, University of British Columbia, \\ 2356 Main Mall, Vancouver, B.C., Canada V6T 1Z4 E-mail: ianc@ece.ubc.ca, jingwa@ece.ubc.ca
}

\begin{abstract}
Recent progress has been made in designing wavelet compression schemes for SAR image data. In this paper, we propose an improvement to the wavelet method based on a subdivision of the image into blocks, so that an optimal bit allocation can be made based on each block's statistics. Unlike single-channel SAR data, polarimetric data has $\mathrm{HH}, \mathrm{VV}$ and $\mathrm{HV}$ channels of radar intensity images, plus the phase differences between them. We examine the information redundancy among these channels, and optimize the block coding scheme across the polarimetric channels and spatial blocks to improve compression performance. Experimental results using SIR-C data show significant improvement in coding efficiency.
\end{abstract}

\section{INTRODUCTION}

Polarimetric data consists of four channels, denoted by $\mathrm{HH}, \mathrm{HV}, \mathrm{VH}$ and VV. The first letter represents the polarization of the transmitted wave channel; the second denotes the received channel. Assuming reciprocity, $\mathrm{HV}$ and $\mathrm{VH}$ can be replaced by $(\mathrm{HV}+\mathrm{VH}) / 2$. Polarimetric data are complex, not only containing three intensity images, but also two phase difference images. Recent studies [1] show that the correlation among them is not high, except for $\mathrm{HH}$ and $\mathrm{VV}$ intensity images. At present, there is no efficient method for decorrelating these images so that they could be better compressed. Because of this, intensity images and phase images are compressed independently, as described in Section II.

At present, most wavelet image compression algorithms [2], [3] are designed for standard test images. Applying such compression schemes to polarimetric data may not lead to ideal results. What hinders the polarimetric data compression is that current compression schemes are not designed to account for the polarimetric data characteristics, such as the speckle phenomena, and significant high frequency components that are highly related to terrain boundaries and textures.

We call the new compression algorithm "Wavelet Packet Embedded Block Coding" or WPEB. It is an evolution of the established "Set Partitioning in Hierarchical Trees" method (SPIHT), which is based on wavelets and is an embedded scheme. Wavelet packet decomposition differs from the standard wavelet analysis by allowing the decomposition of the upper frequency bands as well as the lower ones. In this way, the wavelet packets can be used to achieve more accurate representation of the medium and high frequency information in the SAR images. The block aspect involves dividing the image into blocks so that wavelet decomposition, quantization and coding can be applied to smaller sections of the image, rather than to the whole image at once. In this way, the different statistics of each block can be recognized (especially the activity level), and an optimal bit allocation scheme can be applied to improve the coding efficiency of the whole image. Speckle reduction can also be applied during the encoding/decoding process. The new data compression scheme is based on principles outlined in Section II.

\section{POLARIMETRIC SAR DATA COMPRESSION}

\section{A. Wavelet packet decomposition}

Since polarimetrc data usually have significant middle and high frequency components, as in texture regions, wavelet packets are suitable for polarimetric data compression in that they can effectively preserve these components. In [4], texture analysis for SAR compression is based on subband energy at different decomposition levels. A constant $\mathrm{C}$ is needed as the criterion for wavelet packet decomposition. Energy is defined as:

$$
E=\frac{1}{M N} \sum_{m=1}^{M} \sum_{n=1}^{N}|x(m, n)|^{2}
$$

where $x(m, n)$ is the wavelet coefficient at each subband. If $\mathrm{E}<\mathrm{C} E_{\max }$, wavelet packet decomposition is applied to this subband. In [4], the orthogonal wavelet is used in image decomposition. For the orthogonal wavelet, Parseval's theorem holds so that energy can be a criterion for wavelet decomposition. The disadvantage of using an orthogonal wavelet for image compression is that this type of wavelet cannot achieve linear phase so that symmetric extension is not available to get smooth compression at the block edges. Here, a biorthogonal wavelet is selected to achieve linear phase and symmetrical extension. Since all the images are normalized to zero mean before the discrete wavelet transform (DWT) is applied, variance becomes a valid criterion to decide whether to decompose a given subband further. Normally, the threshold $\mathrm{C}$ should be a value less than 1. As for how to choose $\mathrm{C}$, we suggest that for different types of polarimetric SAR data, $\mathrm{C}$ can be chosen so that significant middle or high frequency components can be preserved at the reconstruction stage.

\section{B. Block coding scheme}

The general idea of embedded block coding is to divide the image into blocks of different sizes. For each block, we apply 
wavelet packet decomposition independently. Then, we encode each block into a binary sequence. Setting up image blocks using a texture criterion is a complicated problem, as an image segmentation algorithm normally takes a long time to execute, and different criteria may produce quite different results. Here we propose a simple blocking algorithm based on quadtree decomposition, where the decomposition levels are variable. The main disadvantage of block coding is artifacts at block edges. In order to reduce this effect, we suggest that the minimum block size be kept to 32 or 64 , which may constrain the maximum decomposition level.

\section{Bit allocation scheme with speckle reduction}

This scheme is designed to assign a different number of bits to each block, which are the results of embedded block coding introduced in section B. Since each encoding design is done at the bit plane, we focus on finding out how many bits should be assigned to each block. If the compression ratio is $\mathrm{R}$ bits per scene, we assign block $\mathrm{n}$ with $\mathrm{Bn}$ bits. The scheme is based on features listed below:

1) Blocks of Importance.

For the block containing important features for classification or detection, more bits should be assigned as done in JPEG 2000. $\{\mathrm{B} 1, \mathrm{~B} 2, \ldots . \mathrm{Bn}\}$ is the bit set to all image blocks, $\{\mathrm{S} 1, \mathrm{~S} 2, \ldots \mathrm{Sn}\}$ is the weight set associated with the bit set representing the weight of importance for each block. If the compression ratio is $\mathrm{R}$ bits per scene, we assign block $\mathrm{n}$ with $\mathrm{Bn}$ bits. Then we have:

$$
\mathrm{R}=\mathrm{S} 1 * \mathrm{~B} 1+\mathrm{S} 2 * \mathrm{~B} 2+\ldots \mathrm{Sn} * \mathrm{Bn}
$$

2) Speckle Reduction.

Wavelet analysis has the advantage that denoising can be easily incorporated into the algorithm, which is useful for SAR speckle reduction. In wavelet transform terminology, speckle reduction is called denoising [5]. The most frequently used denoising methods are called "hard thresholding" or "soft thresholding". Hard thresholding is applied as follows:

(1) Compute DWT: Y=Wy.

(2) Perform thresholding in wavelet domain; $t$ is the threshold with non-negative value.

$$
\hat{X}= \begin{cases}Y, & |Y| \geq t \\ 0, & |Y|<t\end{cases}
$$

(3) Compute inverse DWT: $\hat{x}=W^{-1} \hat{X}$

The difference between soft thresholding and hard thresholding lies in the fact that soft thresholding guarantees that the reconstructed signal is free from spurious oscillations that may result from hard thresholding or Fourier methods. Also, hard thresholding yields better results in terms of $\ell_{2}$ error, which means good signal-noise-ratio for reconstructed images. But in the cases when speckle reduction is the main purpose of utilizing the DWT, soft thresholding is always used.

\section{3) Dynamic Data Range}

SAR data has a large dynamic range, but this varies from block to block. This also applies to the wavelet coefficients. Following rate distortion theory, the compression effect will be improved by varying the bit-allocation scheme, according to the data dynamic range.

\section{EXPERIMENT RESULTS}

The test data is fully polarimetric C-band radar data acquired on April, 1994 during a SIR-C mission. The test area is in the Labrador Sea off the coast of Newfoundland. The multilooked complex data is stored in CEOS format with 10 bytes per pixel. The HH, HV intensity images are obtained from those 10 bytes.

In order to find the parameter $\mathrm{C}$ in wavelet packet decomposition, experiments were done on $\mathrm{HH}$ and $\mathrm{VV}$ intensity images using a three-level $5 / 3$ biorthogonal wavelet transform. For each level decomposition, the ratio of the variance of $\mathrm{LH}, \mathrm{HL}, \mathrm{HH}$ to LL is listed in Table I.

TABLE I. VARIANCE RATIO

\begin{tabular}{|c|c|c|}
\hline & $\begin{array}{c}\text { HH } \\
\text { Intensity }\end{array}$ & $\begin{array}{c}\text { VV } \\
\text { Intensity }\end{array}$ \\
\hline LH1/LL1 & 0.038 & 0.023 \\
\hline HL1/LL1 & 0.036 & 0.019 \\
\hline HH1/LL1 & 0.011 & 0.006 \\
\hline LH2/LL2 & 0.067 & 0.043 \\
\hline HL2/LL2 & 0.062 & 0.030 \\
\hline HH2/LL2 & 0.028 & 0.015 \\
\hline LH3/LL3 & 0.119 & 0.083 \\
\hline HL3/LL3 & 0.090 & 0.043 \\
\hline HH3/LL3 & 0.043 & 0.026 \\
\hline
\end{tabular}

In Table I, some properties can be observed. First, the variance ratio increases as the decomposition level increases. Second, the LL band always contains the most significant information at each level, compared to three other bands at the same level. Generally speaking, for images with large amounts of middle and high frequency components, there is more benefit to applying wavelet packets. In fact, wavelet packets will make wavelet coefficients more compact, so that when using magnitude-ordering algorithms, larger coefficients are coded first and the information at those subbands is preserved much better.

The SNR for the reconstructed $\mathrm{HH}$ intensity image is listed in Table II. WSPIHT is the coding scheme based only on wavelet packets. WPEB is our proposed method based on wavelet packets and block coding. From Table II, it is obvious that WSPIHT outperforms SPIHT at all compression ratios. In addition, WPEB achieves the best result in terms of mean squared error. Figure 1 shows the compression results at $1.2 \mathrm{bpp}$ for the $\mathrm{HH}$ intensity image.

In Figure 1, the WSPIHT compressed image contains more details than the SPIHT compression result because of the 
effect of wavelet packets. The WPEB compression result obtains more details than both SPIHT and WSPIHT by using $64 \times 64$ block size and wavelet packet for each block.

TABLE II. SNR (db) for HH intensity image

\begin{tabular}{|l|l|l|l|}
\hline Bit rate & SPIHT & WSPIHT & WPEB \\
\hline $1.2 \mathrm{bpp}$ & 26.50 & 26.61 & 27.30 \\
\hline $0.6 \mathrm{bpp}$ & 23.40 & 23.64 & 23.71 \\
\hline $0.3 \mathrm{bpp}$ & 21.09 & 21.48 & 21.67 \\
\hline $0.15 \mathrm{bpp}$ & 19.25 & 19.73 & 19.76 \\
\hline
\end{tabular}

\section{CONCLUSIONS}

Wavelet packet decomposition can improve compression performance because the polarimetric SAR data contain significant middle and high frequency components. By utilizing the block coding scheme, image blocks with different statistical properties can be exploited separately based on activity level or energy compaction. The experimental results show that this coding scheme is promising for polarimetric SAR data compression.

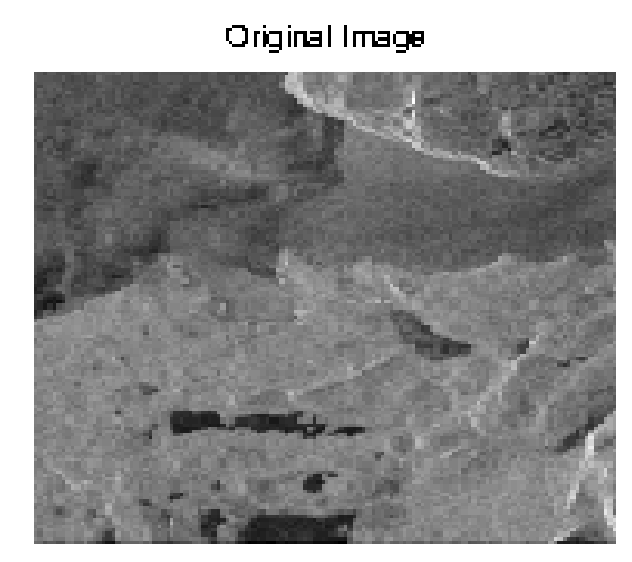

WSPIHT

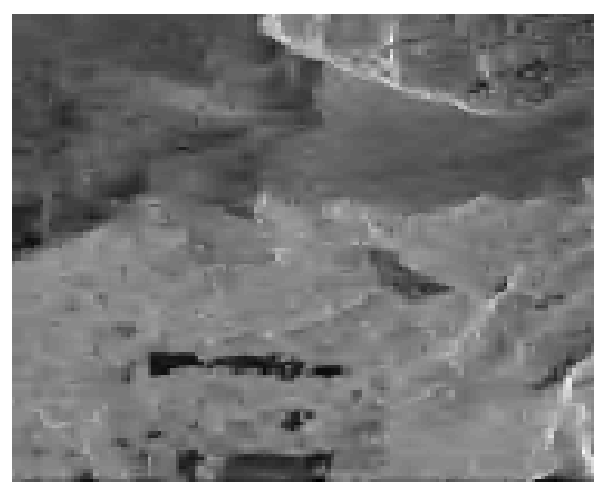

\section{ACKNOWLEDGMENTS}

The authors would like to thank Dr W. A. Pearlman for his valuable comments, and Environment Canada and NASA/JPL for providing data.

\section{REFERENCES}

[1] J-S Lee and K. Hoppel, "Principal Components Transformation of Multifrequency Polarimetric SAR Imagery," IEEE Trans. Geosci. and Remote Sensing, vol. 30, no. 4, pp. 686-696, 1992.

[2] J. M. Shapiro, "Embedded Image Coding Using Zerotrees of Wavelet Coefficients", IEEE Trans. Signal Processing, vol. 41, no. 12, pp. 3445-3462, 1993.

[3] A. Said and W. A. Pearlman, "A New Fast and Efficient Image Codec Based on Set Partitioning in Hierarchical Trees," IEEE Trans. Circuits and Systems for Video Technology, vol. 6, no. 3, pp. 243-250, 1996.

[4] Z. Zeng and I. Cumming, "SAR Image Compression Using a Tree-Structured Wavelet Transform," IEEE Trans. Geosci. and Remote Sensing, vol. 39, no. 3, pp546-552, 2001.

[5] D. Donoho, "De-noising by soft-thresholding," IEEE Trans. Inform. Theory, vol. IT-41, no. 3, pp.613-627, 1995.
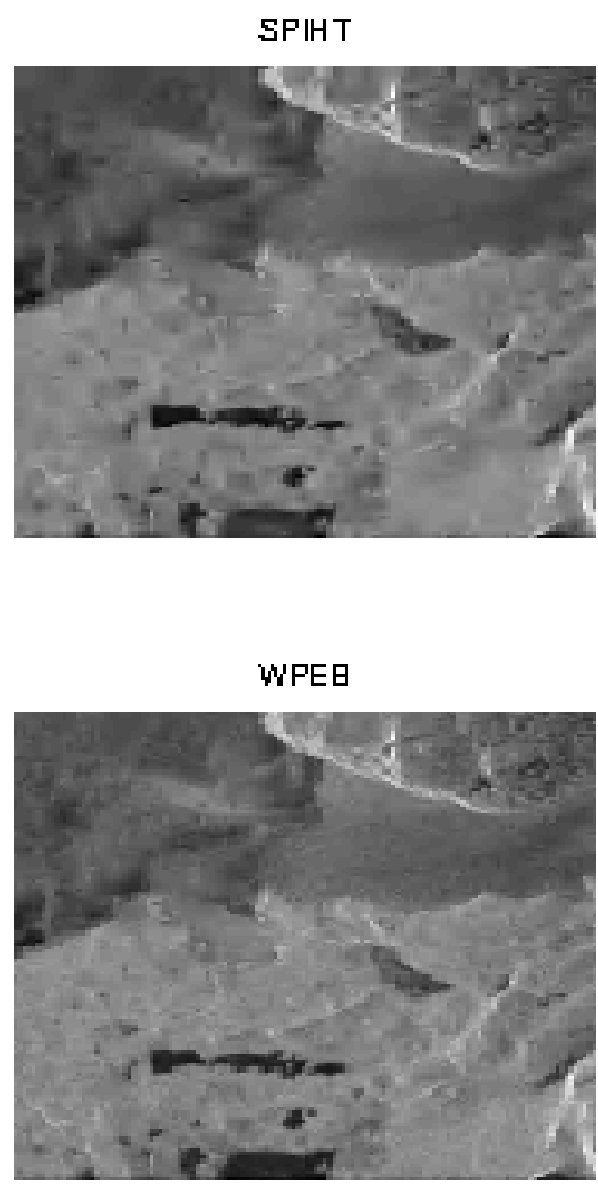

Fig. 1. Compression result at $1.2 \mathrm{bpp}$ for $\mathrm{HH}$ intensity image 